\title{
Husserl critique de la modernité
}

Par LAURENT PERREAU

Université de Picardie Jules Verne - CURAPP-ESS (CNRS-UMR 7319) - Archives

Husserl de Paris (CNRS-UMR 8547)

\section{Introduction}

L'ouvrage ultime de Husserl, La Crise des sciences européennes et la phénoménologie transcendantale ${ }^{1}$, s'inaugure d'une critique des temps modernes (Neuzeit) dont on ne perçoit peut-être plus toutes les singularités, tant il est devenu courant et presque banal de situer dans « la modernité » l'origine des maux qui nous affectent. On ne compte en effet plus les critiques qui, dans des registres variés, s'attachent à décrire la crise de son projet directeur - à quoi Husserl déjà s'adonne - en déplorant tour à tour la faillite de la raison, l'hybris prométhéenne, les ravages du progrès aveugle, la perte des traditions, la dissolution des liens communautaires, la marchandisation de toute chose, le triomphe de la «techno-science», les formes multiples de l'aliénation, etc. Il est en outre difficile d'ignorer le fait que la critique de la modernité a été au cœur des débats, désormais quelque peu datés, autour de la «post-modernité » : elle constitue le présupposé du point de vue "postmoderne » qui s'attache à diagnostiquer les séquelles contemporaines d'une époque à laquelle nous n'appartiendrions déjà plus ${ }^{2}$. Mais il est aussi

${ }^{1}$ Edmund Husserl, Husserliana VI. Die Krisis der europäischen Wissenschaften und die transzendentale Phänomenologie. Eine Einleitung in die phänomenologische Philosophie (1935-1937), W. Biemel (Hrsg.), Den Haag, Martinus Nijhoff, 1954 ; trad. fr. G. Granel et J. Derrida, La Crise des sciences européennes et la phénoménologie transcendantale, Paris, Éditions Gallimard, 1976.

${ }^{2}$ Voir sur ce point Jean-François Lyotard, La Condition post-moderne. Rapport sur le savoir, Paris, Éditions de Minuit, 1979 ; Gianni Vattimo, La Fin de la modernité. 
manifeste que, plus généralement et au-delà de ce foyer de discussion bien identifié, le registre de la critique de la modernité s'impose de manière insidieuse en structurant des pans entiers de la réflexion critique contemporaine, sans que personne ne s'en émeuve, comme s'il était désormais naturel de mobiliser cette catégorie et la perspective généalogique qu'elle implique pour penser, comme d'une position de surplomb, l'époque en général ${ }^{1}$ ou certains problèmes contemporains en particulier ${ }^{2}$. À nos yeux de tard-venus, la critique husserlienne de la science et de la philosophie modernes n'apparaît donc plus que comme une simple déclinaison d'un idéologème désormais largement partagé, une position parmi d'autres au sein d'un espace commun où s'épanouissent, à la faveur d'un concept plurivoque et protéiforme, des interprétations diverses et divergentes d'un moment historique aux contours confus.

Pourtant, la critique husserlienne de la modernité, pour peu qu'on la considère d'un peu plus près, présente suffisamment de reliefs et d'originalité pour qu'on la distingue. On relèvera ainsi que le premier des paradoxes qu'elle présente tient à la nature du singulier diagnostic formulé par Husserl : que «l'humanité européenne » soit en crise à l'époque de Husserl, c'est là un fait indiscutable, mais il est pour le moins étonnant de voir Husserl situer les origines de cette crise dans le dévoiement objectiviste d'une certaine science moderne, en reléguant apparemment à l'arrière-plan toute la dimension

Nihilisme et herméneutique dans la culture post-moderne, Paris, Éditions du Seuil, 1987 et Jürgen Habermas, « La Modernité, un projet inachevé », trad. fr. G. Raulet, dans Critique, $\mathrm{n}^{\circ}$ 413, oct. 1981, p. 950-967.

${ }^{1}$ On mentionnera, entre autres références possibles : Michel Henry, La Barbarie, Paris, PUF, 2004 ; Jürgen Habermas, La Technique et la science comme "idéologie ", trad. fr. J.-R. Ladmiral, Paris, Éditions Gallimard, 1973 ; Hannah Arendt, Condition de l'homme moderne, trad. fr. G. Fradier, Paris, Éditions Pocket, 2001 ; Hans Blumenberg, La Légitimité des Temps Modernes, trad. fr. M. Sagnol et alii, Paris, Éditions Gallimard, 1999 ; Marcel Gauchet, Le Désenchantement du monde. Une Histoire politique de la religion, Paris, Éditions Gallimard, 1994 ou encore Alain Renault, L'Ère de l'individu. Contribution à une histoire de la subjectivité, Paris, Éditions Gallimard, 1991.

${ }^{2}$ On songera par exemple aux réflexions contemporaines en théorie de l'éducation, telle que les conduisent M. Gauchet ou A. Renault, qui à la suite de H. Arendt, ne semblent pas pouvoir s'affranchir du cadre général de la critique de la modernité pour penser les difficultés contemporaines de l'entreprise éducative : Marcel Gauchet, La Démocratie contre elle-même, Paris, Éditions Gallimard, 2002; Alain Renault, La Libération des enfants. Contribution philosophique à une histoire de l'enfance, Paris, Éditions Bayard, 2002. Voir le texte d'Antoine Janvier dans le présent volume. 
politico-sociale de la crise, pourtant manifeste en 1936, et sur laquelle Husserl avait déjà eu l'occasion de méditer dans les fameux articles sur le «Renouveau » pour la revue germano-japonaise Kaizo ${ }^{1}$. Une autre singularité tient sans nul doute à la réaffirmation contre vents et marées d'un certain rationalisme, en quoi la critique husserlienne tranche radicalement par rapport aux critiques sceptiques, pessimistes ou nihilistes de la raison moderne, toutes promptes à dénoncer l'impuissance de la raison à résoudre la crise, quand elles ne font pas de celle-ci l'origine de celle-là. Pour Husserl, l'objectif premier de ses analyses est bien plutôt de trouver les voies d'une restauration de la raison et non de chanter son oraison funèbre, de redonner confiance en la philosophie pour résoudre une crise qui est, en son fond, une crise sceptique $^{2}$. Enfin, une dernière singularité de la critique husserlienne de la modernité tient à la réaffirmation des droits d'une philosophie du sujet que l'on aurait pu croire périmée, comme si un «subjectivisme» renouvelé pouvait encore constituer un remède à la crise : voilà ce qui nous était d'emblée promis avec la découverte de l'ego transcendantal par la méthode de la réduction. Mais, devant l'ampleur de la crise, suffit-il encore de plaider en faveur de l'idéal d'un sujet autonome, ayant pleine conscience de luimême, aspirant en toute chose à la "science » et s'efforçant à une pleine et entière maitrise de ce qu'il fait?

Mais il se pourrait que cette première approche ne soit pas la bonne et qu'elle ne nous permette pas véritablement de cerner la spécificité des réflexions husserliennes. Car ces quelques traits saillants de la critique husserlienne de la modernité ne suffisent peut-être pas pour dissiper l'impression première de banalité : la critique husserlienne n'est, de prime abord, qu'une critique de la modernité parmi d'autres, ce qu'elle était déjà à l'époque si on la resitue dans le contexte de la Kulturkritik des vingt premières années du $\mathrm{XX}^{\mathrm{e}}$ siècle en Allemagne, ce qu'elle est encore plus de nos jours où prolifèrent les discours critiques de la modernité. En outre, on pourra y lire une pure et simple réaffirmation du projet directeur de la modernité, avec lequel elle ne romprait pas véritablement. En un sens, la critique husserlienne de la modernité n'entend pas véritablement s'en excepter ou la dépasser, mais bien la réaliser et l'achever, en la reconduisant

${ }^{1}$ Edmund Husserl, Husserliana XXVII. Aufsätze und Vorträge 1922-1937, T. Nenon et H.-R. Sepp (Hrsg.), Dordrecht/Boston/London, Kluwer, 1989, p. 3-124 ; trad. fr. L. Joumier, Sur le renouveau. Cinq articles, Paris, Librairie philosophique J. Vrin, 2005.

${ }^{2}$ Sur la question du rapport de Husserl au scepticisme, $c f$. Françoise Dastur, « Husserl et le scepticisme », dans Alter, n 11, 2003, p. 13-22. 
à ce qui aurait pu et aurait dû être son sens véritable. Dans cette mesure, la critique husserlienne de la modernité ne peut être comprise comme une contribution phénoménologique au concert des critiques post-modernes de la modernité, mais elle s'expose aussi au reproche d'insuffisance, de naïveté et d'impuissance : est-il vraiment dit que l'on «critique » la modernité si l'on prétend exhiber son sens véritable et rendre possible la réalisation de ce que l'Histoire n'a pas concrétisé ?

Relire les réflexions husserliennes relatives à la modernité exige sans doute que l'on pratique une forme d'épokhè à l'égard des lieux communs de la crisologie de la modernité. Une exégèse charitable devrait donc, avant toute chose, s'autoriser de l'examen attentif des modalités de son traitement phénoménologique. En quoi la crise de la modernité inquiète-t-elle le phénoménologue? Quelle peut être la spécificité du regard phénoménologique porté sur ce moment de l'histoire et quelles sont ses ressources ? Cependant, loin de se résorber, notre malaise grandit immédiatement si l'on se place dans cette perspective rigoureusement phénoménologique et si l'on se remémore quelles peuvent être ses exigences en terme d'exactitude conceptuelle. Car la phénoménologie s'est explicitement présentée comme une tentative de réforme de l'ensemble de notre conceptualité et si cette ambition paraît quelque peu démesurée, on doit au moins lui reconnaître un certain art de l'analytique conceptuelle. Or, sous ce rapport, il est pour le moins étonnant de voir Husserl mobiliser le concept de «modernité » pour en faire la rubrique sous laquelle se développent ses considérations historico-critiques, laissant ainsi opérer une conceptualité qu'il aurait ailleurs dite "naïve », ainsi que ce qu'il faut bien appeler une rhétorique et une dramaturgie, un discours de crise qui semble se nourrir de lui-même. Car lorsqu'il n'est pas employé pour désigner une période rigoureusement définie, comme les historiens ont la sagesse de le faire, en faisant traditionnellement commencer la modernité avec la prise de Constantinople en 1453 et en situant son terme avec la Révolution Française, le concept de modernité court toujours le risque de demeurer une catégorie suspecte d'une philosophie de l'histoire approximative. Le concept de modernité, en raison même de son double caractère polysémique et polémique, est de ceux dont le philosophe raisonnable devrait se garder, ou au moins à l'égard desquels il convient de se montrer d'une extrême prudence. Comment comprendre, dès lors, qu'un philosophe aspirant à l'accomplissement "scientifique » de la philosophie s'accommode d'un instrument conceptuel aussi douteux ? Quelle peut donc être la légitimité phénoménologique du concept de «modernité »? Quel est donc le problème phénoménologique que le recours au terme de «modernité » doit désigner ? 
Or c'est en instruisant cette dernière question, celle de la teneur proprement phénoménologique de la critique de la modernité, que l'on peut espérer restituer sa signification véritable, laquelle ne se conçoit, selon Husserl, que dans le cadre d'une « auto-méditation» (Selbstbesinnung) que le sujet doit conduire sur lui-même. C'est depuis cette perspective particulière, insistante dans la Krisis mais, fort curieusement, souvent négligée par des commentateurs trop pressés d'y découvrir « la » philosophie husserlienne de l'histoire ${ }^{1}$, qu'il convient de reconsidérer le problème. Il apparait alors que la critique de la modernité et la théorie du monde de la vie qui la complète développent aussi et surtout une réflexion critique sur les limites de la subjectivité, dont certains résultats sont paradoxaux. Au rebours de certaines déclarations husserliennes, l'analyse phénoménologique de la crise contemporaine ne promeut ni ne restaure la figure d'un sujet autonome et souverain qui serait en quelque sorte l'instance ultime du Renouveau de la raison. Elle mobilise bien plutôt l'idée d'une subjectivité sédimentée, incarnée, enracinée dans l'histoire et la tradition, dont la puissance est limitée et qui cherche encore à s'orienter dans et par la pensée, par-delà l'opacité des faits ou la confusion de leurs interprétations. Elle consacre aussi paradoxalement une modernité inquiète, encore certaine de son aspiration à la nouveauté et au renouveau, mais incertaine aussi de son succès et consciente des périls qui la menacent.

\section{Les éléments de la crisologie husserlienne de la modernité}

La modernité se dit à l'évidence de plusieurs manières. La pluralité sémantique du concept n'est pas réductible à la diversité des approches disciplinaires (historique, sociologiques, philosophiques, etc.), puisque la question demeure vive de savoir ce qu'il faut exactement entendre sous la catégorie de la modernité au sein même de chaque discipline. En guise de préambule aux considérations qui suivront, nous distinguerons trois usages philosophiques du concept de modernité, en laissant délibérément de côté d'autres déterminations sociologiques, historiques ou littéraires ${ }^{2}$.

\footnotetext{
${ }^{1}$ Voir par exemple Eric Voegelin, « Letter to Alfred Schütz on Edmund Husserl, 17 September 1943 », dans Id., The Collected Works of Eric Voegelin. Volume 6. Anamnesis: On the Theory of History and Politics, David Walsh (ed.), Columbia, University of Missouri Press, 2002, p. 45-61.

${ }^{2}$ On trouvera une présentation synthétique de la polysémie du concept et de ses différentes acceptions disciplinaires dans Yves Vadé, "Présentation », dans Yves
} 
Selon un premier usage, le concept de modernité apparaît comme un opérateur logique qui signale une posture de réflexivité, par laquelle le sujet qui se sait ou se veut «moderne » s'institue comme un sujet qui assume la rupture avec un passé proche ou lointain et la responsabilité de dire ce qui fait la nouveauté de son temps. Il y va donc d'une «mutation de la conscience historique ${ }^{1}$ : pourra se dire « moderne » celui qui s'autorise de la prise de conscience qu'il a de lui-même et corrélativement de son rapport à l'époque. Cet usage est illustré, au tournant du XVIII ${ }^{\mathrm{e}}$ siècle, par la querelle des Anciens et des Modernes, ceux-ci affirmant contre ceux-là le caractère cumulatif du progrès scientifique et la valeur au moins égale de leurs œuvres par rapport à celles de l'Antiquité. Il persiste encore dans l'acception esthétique du terme que scelle Baudelaire ${ }^{2}$ et que Rimbaud érigera en slogan : «Il faut être résolument moderne $»^{3}$. La modernité est donc la nouveauté que l'on réclame pour soi-même, elle est la revendication du sujet qui fonde sa légitimité sur la conscience d'une radicale nouveauté historicosociale. La revendication de modernité est ainsi inséparable de discours modernes qui cultivent sa distinction par rapport à l'ancien.

Un second usage du concept de modernité s'attache à la caractériser et à cerner ses spécificités sociologiques ou historiques, comme le montre exemplairement la théorie wébérienne de la modernisation comme sécularisation et rationalisation. La modernité est un terme qui appelle la définition de son sens. C'est une rubrique générale, le titre d'une description, ou plus précisément de diverses descriptions qui font de ce concept un concept nécessairement plurivoque, de telle sorte qu'il n'est alors plus possible de parler de «la » modernité, mais qu'il convient même sans doute de parler «des» modernités. Le concept de modernité permet alors de coordonner différents aspects (processus ou réalités) qui peuvent être abordés pour euxmêmes, mais qui gagnent aussi en intelligence lorsqu'on les pense synthétiquement — tel est du moins le pari que propose le concept de modernité.

Un dernier usage s'inaugure d'une réflexivité seconde, dérivée, qui met en question les fondements de la conscience de nouveauté dont s'autorise le premier usage. Ce dernier usage suppose le premier, la con-

Vadé (dir.), Ce que modernité veut dire, Tome 1, Bordeaux, Presses Universitaires de Bordeaux, 5, 1994, p. 323.

${ }^{1}$ Gérard Raulet, «Le Concept de modernité », dans Yves Vadé (dir.), Ce que modernité veut dire, op. cit., p. 126.

${ }^{2}$ Charles Baudelaire, «Le Peintre de la vie moderne », dans Id., Cuvres complètes.

Tome 2, Paris, Éditions Gallimard, 1976, p. 695 sq.

${ }^{3}$ Arthur Rimbaud, CEuvres complètes, Paris, Éditions Gallimard, 1972, p. 116.

36 
science de la rupture avec le passé et de l'ouverture de l'avenir, mais il le prend à revers et prend acte des antinomies de la modernité. Selon ce dernier usage, que l'on dira, avec Gérard Raulet, " crisologique »", la modernité ne peut plus être vue comme le résultat de la prise de conscience de la nouveauté d'un moment historique ; elle est ce moment qui institue la crise comme norme implicite de son fonctionnement. Dès lors, la modernité est la contradiction historique, devenue manifeste, entre la foi que l'on accorde à son projet directeur et les désillusions qu'occasionnent les réalités qu'elle engendre. Elle est, indissolublement, " affirmation de la raison dans l'histoire et la crise de la raison dans l'histoire $»^{2}$. La modernité se reflète dans ses propres contradictions et ne peut être appréhendée qu'à travers elles.

Pour Husserl, qui semble hésiter entre les deux derniers usages, la modernité est tout à la fois un thème d'investigation et le nom d'un problème. Cependant l'usage « crisologique » du concept commande l'ensemble de l'analyse : c'est bien parce qu'il y existe une crise contemporaine qu'il faut s'interroger sur le legs de la modernité. Et l'on sait quels sont les divers motifs de la crise en question, qui est tout à la fois crise de la culture, crise des sciences et de la philosophie, crise de l'Europe et, au-delà, du monde occidental. En cela, Husserl n'innove guère puisque son diagnostic relève pour une large part du lieu commun, que son propos concerne la crise des sciences $^{3}$ ou celle de la philosophie ${ }^{4}$. Mais l'originalité de son analyse tient - et c'est aussi sans doute l'une de ses limites - à ce que celle-ci est conçue comme l'expression d'une tendance historique plus lointaine, celle du scepticisme, avec lequel la raison n'aura jamais fini de régler ses comptes :

\footnotetext{
${ }^{1}$ Gérard Raulet, « Le Concept de modernité », art. cit., p. 128.

${ }^{2}$ Yves Vadé, «Présentation », dans Yves Vadé (dir.), Ce que modernité veut dire, op. cit., p 19.

${ }^{3}$ À titre de comparaison, voir par exemple Alfred North Whitehead, Science and the Modern World (1926), New York, The Free Press, 1997. Sur la teneur du diagnostic de crise, $c f$. Pierre Trotignon, Le Cour de la raison. Husserl et la crise du monde moderne, Paris, Éditions Fayard, 1986.

${ }^{4}$ Il y a peut-être ici une lointaine inquiétude qui trouve son origine chez Brentano, lequel s'interrogeait sur «les raisons du découragement dans le domaine de la philosophie » pour conclure que « la philosophie ne peut pas se réjouir d'une grande confiance » (Franz Brentano, «Über die Gründe der Entmutigung auf philosophischem Gebiete » (1874), cité par Léo Freuler, La Crise de la philosophie au XIX ${ }^{e}$ siècle, Paris, Libraire philosophique J. Vrin, 1997, p. 10). Voir aussi Wilhelm Wundt, Über die Aufgabe der Philosophie in der Gegenwart, Leipzig, Engelmann, 1874.
} 
Les combats spirituels authentiques de l'humanité européenne en tant que tels se déroulent comme des combats entre philosophies, savoir: entre les philosophies sceptiques — ou plutôt les non-philosophies, qui ont conservé le thème mais non la tâche — et les philosophies réelles, encore vivantes ${ }^{1}$.

En un sens, la crise dure depuis bien longtemps, même si les symptômes étaient moins évidents, et c'est ce qui légitime la considération rétrospective, sur une longue période, de l'histoire de la philosophie. Le scepticisme est cette négation de la philosophie qui court à travers les siècles et la critique de la modernité n'est en définitive que le moment particulier d'un combat plus long $^{2}$.

La critique husserlienne de la modernité se déploie ainsi, dans un premier temps, à partir de l'examen de deux figures majeures, complémentaires, celle de Galilée dans le domaine des sciences de la nature et celle de Descartes pour la philosophie.

On sait qu'au célèbre paragraphe 9 de la Krisis, Husserl entend montrer comment Galilée procède à l'altération de l'idéal grec de scientificité en promouvant "l'idée d'une totalité d'être rationnelle infinie, systématiquement dominée par une science rationnelle $»^{3}$. Cette scientificité nouvelle, dont on ne voit pas à première vue comment elle pourrait nous conduire à la crise contemporaine, opère via la mathématisation de la nature. La science galiléenne promeut un monde mathématisé, rendu exact et entièrement déterminé. Elle idéalise une nature qui devient elle-même une «multiplicité mathématique ${ }^{4}$. La science galiléenne est donc mathématisation du monde : elle fait descendre l'ordre géométrique dans le monde qui nous entoure, jusqu'à se substituer à lui et devenir la réalité même. Or il apparaît que l'idéalisation qui est à l'œuvre présente trois dimensions, qu'il convient de distinguer.

Une première idéalisation opère au niveau des formes de l'expérience sensible et des corps que celle-ci rencontre dans le monde. À ce monde de la

${ }^{1}$ Hua VI, § 6, p. 13 ; trad. fr., p. 20.

${ }^{2}$ Husserl voit en effet le scepticisme comme une tendance « immortelle » (Edmund Husserl, Hиа VII. Erste Philosophie (1923/1924). Erster Teil: Kritische Ideengeschichte, R. Boehm (Hrsg.), Den Haag, Martinus Nijhoff, 1956, p. 58 ; trad. fr. A. L. Kelkel, Philosophie première (1923/1924). Première partie. Histoire critique des idées, Paris, PUF, 1970, p. 81). Sur le rapport de la philosophie husserlienne au scepticisme, $c f$. Françoise Dastur, "Husserl et le scepticisme », art. cit., p. 13-22.

${ }^{3}$ Hua VI, § 8, p. 19 ; trad. fr., p. 26.

${ }^{4}$ Hua VI, § 8, p. 20 ; trad. fr., p. 27. 
praxis quotidienne, de l'intuition empirique et de la perception sensible, la géométrie - relayée par les techniques de mesure - oppose un monde de formes exactes, rationnellement déterminée et parfaitement définies, un monde de carrés, de triangles et de cercles. Tandis que les choses du monde, dans la perception ordinaire ou dans le jeu de l'imagination, «se tiennent [...] dans une certaine oscillation autour du type pur $»^{1}$, la mathématisation du monde consacre le règne des formes idéales ou "pures ». L'être de la chose est ainsi absolument déterminé «dans une identité absolue $»^{2}$. Avec Galilée, les mathématiques nous livrent un monde exact et «objectif», précisément parce qu'elles donnent immédiatement congé au vague et au subjectif. Les conséquences de cette première idéalisation (la géométrisation du perçu) concernent tous les aspects de notre expérience. Dans le domaine de l'espace, la science nouvelle substitue l'idéalisation géométrique à l'expérience de l'espace, le vécu ${ }^{3}$ prend le pas sur la perception pleine et assurée d'elle-même, comprise comme épreuve des «corps» (au sens du corps vivant ou Leib). L'appréhension du temps elle-même se trouve altérée : la pratique de l'induction remplace l'induction quotidienne, faite d'ajustements partiels et progressifs, par l'intermédiaire des synthèses passives et du réseau des corrélations dans l'espace ${ }^{4}$.

Cependant la radicale nouveauté de la science galiléenne ne s'arrête pas là, puisque cette idéalisation de la chose perçue qui la convertit en pure forme se double d'une seconde idéalisation: celle de la nature conçue comme unique totalité, ensemble de tout ce qui existe, par principe déterminable sur le mode de l'exacte objectivité. Cette idéalisation ontocosmologique conçoit la nature elle-même comme un édifice mathématique ou encore comme un livre écrit en langue mathématique. Le « vrai » monde, la réalité de la réalité, n'est donc plus le monde perçu, mais ce monde de formes pures qu'il recèle en lui et qui ne se découvre qu'à la science. La nouvelle nature n'est plus tant celle de la profusion du vivant que l'idée d'une «totalité infinie d'objectivités idéales » ${ }^{5}$, un a priori qui décide par avance de la possibilité de découvrir, en toute chose, une structure mathématique. On tient là, selon Husserl, le motif secret de toute la modernité :

${ }^{1}$ Hua VI, § 9, p. 22 ; trad. fr., p. 29

${ }^{2}$ Hua VI, § 9, p. 24 ; trad. fr., p. 31.

${ }^{3}$ Hua VI, § 9, p. 22 ; trad. fr., p. 29.

${ }^{4}$ Hua VI, § 9, p. 50 ; trad. fr., p. 58.

${ }^{5}$ Hua VI, § 9, p. 30 ; trad. fr., p. 37. 
La mathématique comme royaume d'une connaissance vraiment objective (et, sous sa direction, la technique), c'était cela qui, pour Galilée, et déjà avant lui, était au foyer de l'intérêt qui mettait en mouvement l'homme "moderne » : l'intérêt pour une connaissance philosophique du monde et pour une praxis rationnelle ${ }^{1}$.

Enfin, un troisième aspect de l'idéalisation à l'œuvre concerne l'intelligibilité du mouvement, désormais compris à partir des interactions causales entre les corps et de la découverte des légalités qui les régissent. Dans le monde de la praxis quotidienne, nous pressentons l'advenue de tel événement sur la base de régularités constatées, par habitude ou encore avec une préscience confuse, sur le mode de l'hypothèse. La science galiléenne présuppose l'existence d'une "causalité universelle idéalisée »" ${ }^{2}$, elle sait calculer les effets produits et prédire avec exactitude ce qui va arriver. Elle garantit l'anticipation exacte, elle est au fondement de la maîtrise technique des mouvements et des interactions et rend ainsi possible une nouvelle technique $^{3}$.

La réinterprétation husserlienne de cette triple idéalisation est célèbre : elle donne lieu à une « substitution [...] par laquelle le monde mathématique des idéalités, qui est une substruction, est pris pour le seul monde réel, celui qui nous est vraiment donné comme perceptible, le monde de l'expérience réelle ou possible : bref, notre monde de la vie quotidien $»^{4}$. Le geste de la modernité est celui d'une découverte (la nature mathématique de la nature) qui recouvre notre perception du monde de ce que Husserl nomme un « vêtement d'idées ou de symboles " ${ }^{5}$. En tant que telle, la scientificité de la science physique n'est pas en cause et on doit bien reconnaître qu'elle est même exemplaire dans sa méthode. Mais cette réussite particulière éclipse le projet d'ensemble qui la sous-tend et l'irrationalité naît ainsi d'une atteinte à l'intégrité du projet original d'une connaissance rationnelle du monde sensible. En un certain sens, pourtant, le monde est bien connu par la science galiléenne, mais il l'est sur un mode unilatéral et objectiviste.

Sur le plan de la philosophie, l'autre figure étudiée est celle de Descartes, qui est à la fois celui qui découvre la subjectivité et manque sa véritable signification. Si la figure de Galilée est au principe de la dérive objectiviste qui affecte, depuis la modernité, notre rapport au monde, celle de

${ }^{1}$ Hua VI, § 9, p. 37 ; trad. fr., p. 44.

${ }^{2}$ Hua VI, § 9, p. 38 ; trad. fr., p. 46.

${ }^{3}$ Hua VI, § 9, p. 53-54; trad. fr., p. 62.

${ }^{4}$ Hua VI, § 9, p. $48-49$; trad. fr., p. 57.

${ }^{5}$ Hua VI, § 9, p. 52 ; trad. fr., p. 60. 
Descartes devient, symétriquement, l'origine d'un subjectivisme qui n'a pas su tenir toutes ses promesses et s'est progressivement dévoyé. Du point de vue de la phénoménologie, l'appréciation de cette figure ne peut donc être qu'ambivalente, puisque Descartes est conçu comme un « précurseur » génial et comme le promoteur d'un «réalisme transcendantal » qui ne pouvait que contrarier durablement l'accès à une authentique philosophie transcendantale. Ainsi, si les Méditations Cartésiennes de 1929 s'employaient à découvrir dans la philosophie cartésienne une inspiration pour la démarche phénoménologique, amplifiant et approfondissant ainsi un recours purement méthodologique déjà à l'œuvre dans les Idées directrices par exemple, elles condamnaient aussi l'orientation durable que Descartes avait imprimée à la philosophie du sujet ${ }^{1}$.

Ce que la Krisis analyse pour sa part est bien plutôt la figure historique du Descartes fondateur de la philosophie moderne ${ }^{2}$, là encore sur un mode très ambivalent. D'un côté, Descartes est celui qui imprime à la philosophie moderne une réorientation décisive, en reformulant le projet d'une philosophie universelle et en systématisant les moyens de son accomplissement. Il est ainsi à l'origine d'un motif téléologique qui détermine de manière sous-jacente toute l'histoire de la philosophie moderne, le motif d'une philosophie fondationnaliste qui se donne en réponse à une crise sceptique de longue durée. Descartes fait d'une simple idée une « idée historique $»^{3}$ en lui donnant sa première concrétisation historique. Tout en participant à l'essor du rationalisme physiciste et en contribuant à la nouvelle philosophie de la nature, Descartes est aussi celui qui promeut un certain nombre de motifs philosophiques qui devaient, à terme, amener la philosophie à entrer en contradiction ouverte avec la science objectiviste et à ruiner les fondements de sa Weltanschauung ${ }^{4}$.

Cependant, d'autre part, aux yeux de Husserl, Descartes est aussi l'une des origines de crise de la modernité, puisqu'il est l'instigateur d'un « dualisme » qui tire en réalité toutes les conséquences de l'objectivisme de la science nouvelle. Tout en restant fidèle à l'esprit de la révolution galiléenne, Descartes opère une distinction entre le domaine de la nature,

${ }^{1}$ Edmund Husserl, Husserliana I. Cartesianische Meditationen und Pariser Vorträge (1929), S. Strasser (Hrsg.), Den Haag, Martinus Nijhoff, 1950, § 10, p. 63 sq. ; trad. fr. M. de Launay, Méditations Cartésiennes et les conférences de Paris, Paris, PUF, 1994, p. $67 \mathrm{sq}$.

${ }^{2}$ Les mentions abondent : Hua VI, p. 18, 74-80, 83, 392, 424 ; trad. fr., p. 25, 85-91, 94, 433, 469 .

${ }^{3}$ Hua VI, § 16, p. 75 ; trad. fr., p. 86.

${ }^{4}$ Hua VI, § 16, p. 75 ; trad. fr., p. 86. 
réservé à l'investigation de la nouvelle science, et celui de l'esprit, de l'âme ou de la subjectivité, laquelle est au principe de «l'opposition moderne entre l'objectivisme physiciste et le subjectivisme transcendantal ${ }^{1}$. Descartes achève ce qui était déjà présent chez Galilée, lequel a promu « une nouvelle idée de la nature en tant que monde-de-corps séparé, réellement et théoriquement clos sur lui-même $»^{2}$. Cette abstraction impliquait la conception de l'âme ou psyché définie comme principe de la vie animale ou humaine, distincte du reste de la nature. Le dualisme corps/esprit suppose donc rien de moins qu'un nouveau partage du monde :

Le monde se dissocie pour ainsi dire en deux mondes : nature et monde-dupsychologique dont le second à vrai dire ne s'élève pas à la consistance d'un être-monde, à cause de la façon dont il est relié à la nature ${ }^{3}$.

Cependant, la «psychologie» nouvelle qui semble ainsi appelée par la physique galiléenne se situe encore dans la dépendance de celle-ci, puisqu'elle se réalise sur un mode naturaliste. Le second tort de Descartes aura été de «psychologiser» le retour à l'ego en commettant le contresens fondamental du « réalisme transcendantal» qui le convertit en res cogitans, c'est-à-dire en une chose du monde ${ }^{4}$. Descartes fonde la psychologie moderne tout en rendant proprement incompréhensibles les opérations de la subjectivité, en donnant ainsi bien malgré lui de nouveaux motifs au scepticisme.

\section{La modernité, cet autre historique de la phénoménologie}

La phénoménologie husserlienne développe donc une critique de la modernité qui, comme tant d'autres, trouve sa spécificité dans la mise en exergue de certaines caractéristiques tenues pour déterminantes de toute une époque. Soit, dans le cas présent, l'essor des sciences de la nature, la mathématisation de l'ensemble du réel, le développement d'une psychologie aveugle à l'activité constituante de la conscience. Cette critique se focalise sur l'examen de deux figures historico-philosophiques majeures, Galilée et Descartes, hissées au rang de figures quasi-mythiques représentant à elles seules les mouvements novateurs qui animent science et philosophie.

${ }^{1}$ Hua VI, § 8, p. 18 ; trad. fr., p. 25.

${ }^{2}$ Hua VI, § 9, p. 61 ; trad. fr., p. 70.

${ }^{3}$ Idem.

${ }^{4}$ Hua VI, § 18, p. 82 ; trad. fr., p. 93. 
Si l'exégèse husserlienne a souvent souligné l'importance du moment galiléen ${ }^{1}$, il faut pourtant insister sur la complémentarité de ces deux figures. Chacune d'elle représente une tendance qui s'oppose apparemment à l'autre : l'objectivisme scientifique pour Galilée, le subjectivisme philosophique pour Descartes, comme si le défi de la modernité résidait tout entier dans l'écart et l'approfondissement respectif de deux tendances divergentes, l'une étant proprement fascinée par l'objectivité mathématique, l'autre inaugurant l'exploration «transcendantale » du sujet sans parvenir à la mener à bien. Dans cette distribution, il est patent que le partage n'est toutefois pas égal puisqu'en définitive le subjectivisme cartésien ne vient pas contredire l'objectivisme scientifique, mais demeure parfaitement compatible avec lui, dans la mesure où il adhère à ses présupposés fondamentaux, voire s'inspire de sa méthode. Il n'en reste pas moins qu'une opposition se trouve ainsi instituée, dont nous sommes encore largement les héritiers, et qui ne pouvait selon Husserl que nous conduire à la crise contemporaine.

On pourra aisément remarquer que, dans cette double lecture de la philosophie galiléo-cartésienne, il y a en définitive beaucoup de Husserl, comme si la phénoménologie ne faisait autre chose que se chercher ellemême à travers ces figures historiques, projetant son ombre sur l'histoire de la philosophie pour mieux faire ressortir quelques moments saillants. Comment ne pas voir en effet, dans l'identification de cette divergence fondamentale entre objectivisme et subjectivisme (ou plutôt dans cette forme «manquée » de subjectivisme qui est celle de la philosophie cartésienne), une inversion radicale de ce que nous propose la phénoménologie ? Poussons plus loin cette lecture : la modernité, telle que la définit Husserl, n'est-elle pas en définitive cet autre historique de la phénoménologie, puisque cette période présente à la fois une alternative historique à une phénoménologie non encore advenue et, par suite, son image défaite ou à tout le moins inachevée? La critique de la modernité est en définitive celle d'une possibilité qui a trouvé à se réaliser dans l'Histoire, possibilité qui ne se comprend elle-même qu'à partir de la possibilité de la phénoménologie, exhibée en retour comme possibilité immémoriale (puisqu'en un sens, elle était bien, selon Husserl, de tout temps, possible). Elle est aussi, sur un double mode généalogique et téléologique, ce qui nous permet de comprendre les raisons des difficultés de l'accès à la phénoménologie et de sa diffusion, mais aussi de rendre compte de sa nécessité actuelle comme réponse à la crise.

${ }^{1}$ On recommandera l'ouvrage de François de Gandt, Husserl et Galilée. Sur la crise des sciences européennes, Paris, Libraire philosophique J. Vrin, 2004. 
Car la phénoménologie représente bien cette tentative inédite pour faire tenir ensemble, en un certain sens qui n'est certes plus celui de la modernité, "objectivisme» et "subjectivisme ». Husserl rappelle très clairement, au fameux paragraphe 48 de la Krisis, que la tâche de la phénoménologie n'est autre que celle de la mise au jour et de l'exploration de «l'a priori corrélationnel universel de l'objet d'expérience et de ses modes de données ${ }^{1}$, soit la description et l'analyse des structures de l'intentionnalité, cette fameuse caractéristique de la conscience en vertu de laquelle le sujet a, précisément, conscience d'objet. La découverte phénoménologique de l'intentionnalité, de «ce champ infini de l'a priori de la conscience, auquel on n'a jamais fait droit dans ce qu'il a de propre, qui n'a même jamais été proprement $\mathrm{vu} »^{2}$, représente bien, pour Husserl, une solution à l'énigme fondamentale de la connaissance, dans la mesure où elle restitue à la subjectivité la part qui lui revient dans la genèse et le déroulement de toute expérience. Le correctif historique que la phénoménologie entend apporter à la modernité tient à cette réconciliation de l'objectivisme et du subjectivisme que seule la théorie de l'intentionnalité et la pratique effective de l'analyse phénoménologique de la conscience autorise.

La phénoménologie prétend donc découvrir ce que le projet moderne n'avait cessé de s'obstiner à manquer, nous offrant ainsi la possibilité de rectifier un dévoiement et de dépasser un moment d'égarement. En effet, la modernité galiléo-cartésienne promeut un rapport au monde et une théorie de ce rapport qui est radicalement opposée à la conception phénoménologique de la corrélation intentionnelle. Elle creuse l'écart entre le sujet et l'objet et nous rend durablement aveugle au fonctionnement véritable de la subjectivité. La modernité est, de ce point de vue, bien plus qu'une simple alternative, elle est alors l'obstacle épistémologique qui entrave l'accès à la phénoménologie, ce qui conduira d'ailleurs Husserl à désavouer le cheminement méthodologique frayé dans les Idées directrices... de 1913 et à reconnaître sa préférence pour la voie « historique » ouverte dans la Krisis, jugée «plus principielle et plus systématique $»^{3}$ : ce que la modernité consacre et

${ }^{1}$ Hua VI, § 48, p. 169 ; trad. fr., p. 189.

${ }^{2}$ Edmund Husserl, Husserliana III/1. Ideen zu einer reinen Phänomenologie und phänomenologischen Philosophie. Erstes Buch: Allgemeine Einführungin die reine Phänomenologie 1, K. Schuhmann (Hrsg.), Den Haag, Martinus Nijhoff, 1977, § 63, p. 135 .

${ }^{3}$ Edmund Husserl, Hua XXIX. Die Krisis der europäischen Wissenschaften und die transzendentale Phänomenologie. Ergänzungsband. Texte aus dem Nachlass 19341937, Reinhold N. Smid (Hrsg.), Dordrecht/Boston/London, Kluwer Academic Publishers, 1993, texte $n^{\circ} 34$, p. 426. 
théorise en définitive, c'est l'attitude naturelle, ce rapport spontané de la conscience au monde qui vit dans la fascination de l'objet, « cette attitude de l'existence humaine naturelle qui, non pas de manière contingente mais par essence, est toujours la première, cette attitude qui, dans toute l'histoire de cette existence, dans la vie comme la science, n'avait jamais été interrompue $»^{1}$. Attitude naïve et naturelle, qui vit dans l'immédiatement de la donation, «validation muette $»^{2}$ qui pose l'existence objective de l'étant sans pouvoir thématiser cette position, qui est au fondement de toutes nos activités pratiques et théoriques. L'attitude naturelle est au principe d'un "réalisme objectiviste » qui est au fondement de la démarche galiléenne et que celle-ci nourrit en retour. Et si la phénoménologie, pour révéler la corrélation intentionnelle, doit destituer l'attitude naturelle, elle ne peut donc se concevoir, historiquement, que comme une rupture radicale par rapport à la modernité. La modernité est ce moment de l'histoire de la philosophie où la théorie de l'intentionnalité s'est trouvée manquée et où toutes les conditions ont été réunies, de manière durable, pour que celle-ci ne soit pas mise au jour. La méthodologie de la réduction ne peut alors plus être conçue sur un mode strictement cartésien, puisqu'elle doit s'inaugurer d'une réflexion « en retour » sur ses conditions de possibilité historiques.

Dans le même temps, l'événement que constitue l'irruption de la phénoménologie à l'époque contemporaine ne peut pas être compris comme une pure sortie de la modernité. Si, comme Husserl le souligne dès l'Introduction du premier livre des Ideen de 1913, «la phénoménologie pure» est «une science essentiellement nouvelle ${ }^{3}$, elle est aussi «la secrète aspiration de toute la philosophie moderne ${ }^{4}$. L'apparition de la phénoménologie est une nouveauté radicale, mais elle est entièrement préparée par la modernité, qu'elle achève à sa manière. La phénoménologie prétend satisfaire l'exigence d'une scientificité accomplie et désigner la seule instance susceptible d'y pourvoir, la subjectivité transcendantale. En quoi Descartes est bien aussi, rétrospectivement, « le génie fondateur originel de la philosophie moderne dans son ensemble ${ }^{5}$ puisqu'il détermine

\footnotetext{
${ }^{1}$ Hua VI, § 41, p. 154 ; trad. fr., p. 172.

${ }^{2}$ Hua VI, $\S 40$, p. $152 ;$ p. 170.

${ }^{3}$ Hua III/1, p. 3.

${ }_{5}^{4}$ Hua III $/ 1, \S 62$, p. 133.

${ }^{5}$ Hua VI, § 16, p. 75 ; trad. fr., p. 85. Voir aussi Hua VI, appendice X (1936), p. 425; trad. fr., p. 469 ; Hua VII, p. 63; trad. fr., p. 89 ; Edmund Husserl, Husserliana XVII. Formale und transzendentale Logik. Versuch einer Kritik der logischen Vernunft. Mit ergänzenden Texten, P. Janssen (Hrsg.), Den Haag, Martinus Nijhoff, 1974,
} 
l'orientation subjective à même de satisfaire à l'exigence qui définit la philosophie comme telle. C'est pourquoi «ce n'est pas seulement le caractère fondamental de la philosophie moderne, mais aussi celui de toute philosophie future, qui est par là déterminé à partir de Descartes » ${ }^{1}$. En un sens, la phénoménologie est bien cet accomplissement philosophique que la modernité attendait.

Mais à ce compte, on ne voit pas comment on pourrait épargner à cette philosophie husserlienne de l'histoire le reproche d'une surinterprétation sélective, dont l'indice le plus manifeste tient sans doute à l'obsession de se donner des "précurseurs », selon un usage bien commode de l'histoire dont Canguilhem a fait la critique que l'on sait ${ }^{2}$. Il sera également bien difficile de se départir de l'impression que l'on ne cesse de retrouver dans l'histoire les signes annonciateurs d'une phénoménologie qui, seule, pourrait livrer la signification de l'histoire, comme si l'histoire ne trouvait jamais son « sens » véritable que depuis la phénoménologie, comme si entrer en phénoménologie, c'était en définitive n'en plus pouvoir sortir et se condamner à une considération de l'histoire qui ne pourrait que s'achever en «poésie » ou en mythologie.

\section{L'auto-méditation de la modernité}

La question qui ne se pose donc inévitablement, au terme de cette critique de la modernité, est formulée par Husserl lui-même dans l'appendice XXVIII au paragraphe 73 de la Krisis : «Quel est ici le besoin de l'histoire ?»" ${ }^{3}$. Quel besoin a-t-on de penser l'événement de l'apparition de la phénoménologie sur fond de modernité ? Si cet événement doit avoir l'importance que Husserl lui accorde, ne peut-on s'épargner ce long détour critique ? Si la phénoménologie est, de manière si éclatante, le remède aux maux de l'époque, pourquoi ne s'impose-t-elle pas d'elle-même?

Mais précisément, les choses ne sont jamais gagnées d'avance et ce que la critique de la modernité nous enseigne, c'est aussi et surtout que la

p. 235 ; trad. fr. S. Bachelard, Logique formelle et logique transcendantale. Essai d'une critique de la raison logique, Paris, PUF, 1965, p. 306.

${ }^{1}$ Edmund Husserl, Husserliana XXXV. Einleitung in die Philosophie. Vorlesungen 1922/1923, B. Goosens (Hrsg.), Dordrecht/Boston/London, Kluwer, 2002, p. 313 ; trad. fr. A. Mazzù, Annales de phénoménologie, vol. 2, 2003, p. 163.

${ }^{2}$ Georges Canguilhem, Études d'histoire et de philosophie des sciences concernant les vivants et la vie, Paris, Librairie philosophique J. Vrin, 1990, p. 20 sq.

${ }^{3}$ Hua VI, appendice XXVIII, p. 508 ; trad. fr., p. 563. 
crise est en nous. La crise de la modernité est nécessairement une crise du sujet moderne, en tant qu'il est le produit de cette modernité et qu'il entretient un rapport particulier à son projet directeur. C'est en ce sens que la «présentification dans le souvenir historique » n'est «encore rien d'autre qu'une présentification de nous-mêmes ${ }^{1}$. Chez Husserl, la crisologie opère prioritairement depuis le registre de l'égologie : «Une réflexion historique en retour, telle que celle dont nous parlons, est ainsi véritablement une très profonde auto-méditation sur la compréhension de soi-même $»^{2}$. Puisque les idées agissent comme des "forces instinctives »" la considération téléologique de l'histoire va donc nécessairement de pair avec une "automéditation » que le sujet conduit sur lui-même, cette Selbstbesinnung dont le paragraphe 15 de la Krisis précise brièvement, mais de manière décisive, la méthodologie ${ }^{4}$. Cette «auto-méditation» est la saisie consciente de l'unité téléologique foncière de l'histoire de la philosophie et elle est le site véritable, premier et ultime, de toutes les réflexions relatives à la modernité.

Cette «auto-méditation» se distingue de la réflexion opérant sur un mode cartésien, par laquelle le sujet revient sur lui-même en se révélant à luimême tout ce qu'il peut tirer de lui-même. La Selbstbesinnung opère bien plutôt dans le registre de la passivité, en faisant retour sur une expérience sédimentée. Car ce qui est à «méditer» n'est pas tant le sujet lui-même comme instance responsable de la constitution de toute objectité, que le sens de l'expérience historique et personnelle. L'auto-méditation découvre donc ce qui gît dans le sujet, l'histoire incorporée et oubliée qui agit le sujet, cette téléologie descendue dans l'intimité qu'elle nous donne l'occasion de nous réapproprier. La téléologie qui oriente l'histoire découvre son fondement ultime dans la structure téléologique de la subjectivité :

Il convient donc que nous donnions la compréhension de la téléologie dans le devenir historique de la philosophie, en particulier de celle des modernes, et du même coup que nous nous mettions au clair sur nous-mêmes en tant que

${ }^{1}$ Hua VI, appendice V, p. 392 ; trad. fr., p. 433.

${ }^{2}$ Hua VI, § 15, p. 73 ; trad. fr., p. 83.

${ }^{3}$ Hua VI, § 16, p. 75 ; trad. fr., p. 86.

${ }^{4}$ Hua VI, § 15, p. 71-74; trad. fr., p. 81-85. Signalons également le texte ${ }^{\circ} 32$ de Edmund Husserl, Husserliana XXIX. Die Krisis der europäischen Wissenschaften und die transzendentale Phänomenologie. Ergänzungsband. Texte aus dem Nachlass 1934-1937, R. N. Smid (Hrsg.), Dordrecht/Boston/London, Kluwer, 1993, p. 362420 ; trad. fr. par nos soins, Sur l'histoire de la philosophie. Choix de textes, Paris, Librairie philosophique J. Vrin, 2014. 
nous sommes les porteurs de cette téléologie et que notre dessein personnel est de contribuer à l'accomplir ${ }^{1}$.

L'auto-méditation s'inaugure de la prise de conscience de cette ou ces téléologie(s), en considérant les moments historiques décisifs de leur formulation. Elle passe par la «compréhension critique de l'unité d'ensemble de l'histoire $»^{2}$, mais dépasse celle-ci vers la mise au jour d'une « tâche », c'està-dire d'un projet légué par l'histoire et que le sujet se réapproprie. Elle aboutit, idéalement, au choix déterminé, et en l'occurrence à cette réponse à la crise de la modernité qui réside, selon Husserl, dans l'assomption personnelle de la philosophie comme tâche et dans un renouveau de la raison. On voit quel est le prix que Husserl est prêt à payer, celui d'une philosophie de l'esprit transhistorique, d'une "volonté » s'affirmant dans l'histoire, animant celle-ci d'une téléologie secrète. La phénoménologie pousse ainsi la théorie de l'intentionnalité à ses limites, puisque la vie intentionnelle se confond en définitive avec une intention transhistorique qui se communique à nous. C'est aussi reconnaître qu'il y a une normativité historique qui décide du sens de l'expérience, non pas certes au sens d'un déterminisme grossier, mais plutôt en ce sens que la vie intentionnelle présente bien, de part en part, une dimension sociale et historique qui est le double produit de la passivité et de la générativité, de ce que Husserl nomme encore la «sédimentation traditionnelle $»^{3}$.

Dans ces circonstances, la théorie de la modernité ne se déploie plus seulement dans la perspective d'une philosophie de l'histoire soucieuse du «fait» et c'est en cela que réside la véritable singularité des analyses husserliennes, aussi étranges qu'originales à cet égard. La contemplation de la modernité inspire une réflexion d'ordre éthique, un retour sur soi du sujet qui découvre l'intention historique dont il est le vecteur, le sens de l'histoire qui décide de son historicité. Elle devient, en ce sens, l'occasion d'une transformation de soi qui donne accès à une forme bien particulière de responsabilité. La théorie de la modernité est ainsi une tentative originale pour fonder la responsabilité individuelle à partir de l'histoire : la révélation des tendances qui œuvrent dans l'histoire de la philosophie fournit l'occasion d'une reprise active, pleinement assumée de la critique rationnelle. La fin de

\footnotetext{
${ }^{1}$ Hua VI, $\S 15$, p. 71 ; trad. fr., p. 81.

${ }^{2}$ Hua VI, § 15, p. 72 ; trad. fr., p. 82.

${ }^{3}$ Hua VI, $\S 15$, p. 72 ; trad. fr., p. 83. Sur la question de la générativité, nous renvoyons le lecteur aux analyses développées dans Laurent Perreau, Le Monde social selon Husserl, Dordrecht/Boston/London/New York, Springer, 2013.
} 
la Conférence de Vienne formule dès lors cette alternative où le choix s'impose en quelque sorte de lui-même :

La crise de l'existence européenne ne peut avoir que deux issues : ou bien le déclin de l'Europe devenue étrangère à son propre sens rationnel de la vie, la chute dans la haine spirituelle et la barbarie, ou bien la renaissance de l'Europe à partir de l'esprit de la philosophie, grâce à un héroïsme de la raison qui surmonte définitivement le naturalisme ${ }^{1}$.

Il pourra être tentant de voir dans cet « héroïsme de la raison » une forme de volontarisme quelque peu grandiloquent ${ }^{2}$ et le retour forcé et naïf d'un sujet auto-suffisant, auto-subsistant qui serait l'incarnation d'une raison pourvoyeuse d'une connaissance de soi et du monde, d'une maitrise pleine et entière de soi. Mais il est sans doute plus juste d'y découvrir, c'est du moins l'interprétation qui aura notre préférence, un sujet sensible à la précarité de l'aspiration à la raison, un sujet conscient de sa finitude historique, vivant encore et toujours le malaise de la modernité, débrouillant l'expérience sédimentée de l'histoire. Paradoxalement, l'aspiration à la connaissance de soi, que célébraient encore avec force les dernières pages des Méditations Cartésiennes, nous montre désormais que nous ne nous connaissons pas nous-même et plus précisément que nous n'en aurons jamais fini avec cette méconnaissance. La philosophie - ou le renouveau de la raison - ne peut être, en ce sens, qu'une tâche infinie, qui n'est certes pas une désespérante impossibilité, mais qu'il ne nous est pas non plus possible de réaliser dans les circonstances que nous connaissons et dans les limites qui sont les nôtres.

\section{Conclusion}

Le concept de modernité demeure une catégorie précieuse pour cerner les spécificités d'une période historique et mettre en perspective l'époque contemporaine. Mais cet usage que l'on pourra dire encore relativement «historien» est constamment doublé, chez Husserl, de la méditation personnelle de la crise de la modernité, travail que le philosophe conduit sur lui-même, où il s'agit en définitive de faire le départ entre différentes motivations léguées par la tradition. On voit bien quel est l'effet de ce

${ }^{1}$ Hua VI, appendice XIII, p. 347 ; trad. fr., p. 382.

${ }^{2}$ Sur la discussion du «volontarisme» husserlien, cf. R. Philip Buckley, Husserl, Heidegger and the Crisis of Philosophical Responsability, Dordrecht/Boston/London, Kluwer, p. $131 s q$. 
dédoublement de la réflexion : la modernité ne paraît alors plus susceptible d'une définition rigoureuse, ce que Husserl lui-même semble reconnaître, lorsqu'il concède, dans un appendice célèbre à la Krisis, pratiquer une sorte de «poésie» de l'histoire ${ }^{1}$. La «modernité» est l'occasion d'une automéditation, la téléologie de l'histoire intentionnelle se confondant in fine avec la réflexion que le sujet mène sur le "choix» d'une raison en crise. Réclamée par le constat de la «crise radicale de la vie dans l'humanité européenne $»^{2}$, l'auto-méditation fait de cette crise une contradiction personnelle, intime, qui écartèle le sujet méditant entre le choix résolu de la raison et la tentation du renoncement. En ce sens, la considération husserlienne de la modernité est bien discutable, mais peut-être pas au sens où on l'entend ordinairement — c'est-à-dire au sens où l'on pourrait bien contester certains aspects des analyses husserliennes relatives à tel ou tel aspect de la crise ou à telle ou telle caractéristique de la « modernité », ce qui nous situe encore dans le champ de la philosophie de l'histoire. Elle est discutable au sens où il faut désormais débattre de son logos intime, d'une histoire ressaisie au niveau de la vie intentionnelle. Car tout l'effort de Husserl consiste précisément à nous donner les moyens de rediscuter ce sur quoi il n'y avait plus discussion et à replacer le sujet en situation de choix devant l'histoire.

Sous la rubrique de la modernité, le fait historique est ainsi reconverti pour devenir une donnée livrée au régime de la Selbstbesinnung. Tout nous signale donc que l'on a affaire à une pure interprétation philosophique d'une époque si longue que sa consistance historique ne peut plus, précisément, qu'être sujette à interprétation. En ce sens, Husserl ne cesse de mettre à l'épreuve la portée et les limites du concept de modernité, en nous invitant à une réflexivité critique concernant son usage légitime et ses mésusages. À l'heure où abondent les discours de surplomb prétendant nous dire avec assurance ce qu'est la modernité, ce qu'il faut en penser et comment et pourquoi il faudrait en sortir, il est bon de relire ces textes husserliens qui pratiquent, en un certain sens, une forme de crisologie encore inquiète des ressources conceptuelles qu'elle mobilise.

${ }^{1}$ Hua VI, appendice XXVIII, p. 512 ; trad. fr., p. 567. Voir également l'article de Jocelyn Benoist, "L'histoire en poème», dans Recherches husserliennes, vol. 9, 1998, p. 91-112.

${ }^{2}$ Hua VI, p. 1 ; trad. fr., p. 7. 